\title{
Érase una vez en el este: retos de conservación de la biodiversidad en el amanecer económico de un país poscomunista
}

\author{
Ladislav Miko ${ }^{1}$ y Jan Plesnik ${ }^{2}$ \\ Observa en profundidad la naturaleza, \\ y entonces entenderás todo mejor
}

Albert Einstein

Sumario: I. INTRODUCCIÓN. II. HISTORIA: UNA LARGA TRADICIÓN DE CONSERVACIÓN DE LA NATURALEZA. III. LA LEGISLACIÓN COMO PIEDRA ANGULAR DE UNA CONSERVACIÓN DE LA NATURALEZA Y PROTECCIÓN DEL PAISAJE EFICACES TRAS LA REVOLUCIÓN DE TERCIOPELO. IV. INCORPORACIÓN A LA UNIÓN EUROPEA, NATURA 2000 Y TENDENCIAS ACTUALES EN MATERIA DE CONSERVACIÓN DE LA NATURALEZA. V. ELEMENTOS PRINCIPALES DE LA POLÍTICA ACTUAL DE CONSERVACIÓN DE LA NATURALEZA. 1. Búsqueda de prioridades estratégicas. 2. Áreas protegidas (AAPP). 3. Paisajes no reservados y protección del paisaje. 4. Enfoque participativo. 5. Interrelación política-ciencia y evaluación de la biodiversidad. 6. Información, educación y concienciación pública (CEPA). VI. NATURALEZA Y CAMBIO CLIMÁTICO COMO NUEVO RETO: UNA HISTORIA DE MITIGACIÓN Y ADAPTACIÓN. 1. Política nacional de mitigación y adaptación al cambio climático. 2. Adaptación basada en los ecosistemas. 3. Gestión adaptativa. VII. POLÍTICA DE CONSERVACIÓN DE LA NATURALEZA Y RETOS EN LA PRÓXIMA ETAPA: ¿RECUERDOS DEL FUTURO? VIII. BIBLIOGRAFÍA. ANEXO 1. BIODIVERSIDAD: ¿ENIGMA O TRIVIALIDAD? ANEXO 2. LA NATURALEZA Y EL PAISAJE EN LA REPÚBLICA CHECA SE PRESENTAN BREVEMENTE.

1 Ministro, Ministerio de Medio Ambiente de la República Checa, Vrsovicka 65, CZ-100 10 Praha 10.

2 Asesor de Dirección, Agencia para la Conservación de la Naturaleza y la Protección del Paisaje de la República Checa, Nuselska 39, CZ-140 oo Praha 4. 


\section{RESUMEN}

A pesar del insatisfactorio estado en que en general se encuentra la naturaleza, claro ejemplo de lo cual es el Triángulo Negro, en la República Checa se han conservado partes valiosas de la naturaleza en un estado relativamente bueno, o que permite el restablecimiento de los procesos naturales básicos. Los cambios políticos, económicos y sociales acaecidos en la antigua Checoslovaquia después de la Revolución de Terciopelo de 1989 atendieron de manera especial a la protección del medio ambiente, incluidas la conservación de la naturaleza y la protección del paisaje. La nueva normativa sobre conservación de la naturaleza se basa en los enfoques actuales en materia de conservación de la biodiversidad, habiéndose creado la Agencia para la Conservación de la Naturaleza y protección del Paisaje de la República Checa. El elemento clave de la legislación es la Ley N.`114/1992 sobre Protección de la Naturaleza y del Paisaje, modificada en 2004 para transponer la normativa comunitaria relativa a la conservación de la naturaleza. Se describe el desarrollo de la política de conservación de la naturaleza y protección del paisaje en la República Checa. Los autores abordan, entre otras, cuestiones como la planificación de la conservación, las zonas protegidas, el paisaje no protegido y la protección del paisaje, el enfoque participativo, la interrelación ciencia-política y la evaluación de la biodiversidad, la información, educación y concienciación pública, la adaptación al cambio climático basada en los ecosistemas y la gestión adaptativa.

\section{PALABRAS CLAVE}

Naturaleza, paisaje, conservación de la naturaleza, protección del paisaje, gestión de la biodiversidad, política de conservación de la naturaleza, República Checa, Unión Europea.

\section{INTRODUCCIÓN}

La República Checa es un país poscomunista, que desde 2004 es Estado Miembro de la Unión Europea (UE). Las características básicas del patrimonio natural y paisajístico del país se encuentran sintetizadas en el Anexo 1. En el último siglo, aproximadamente, la República Checa ha conocido diversas formas de organización y administración. Hasta 1918 fue una de las partes más desarrolladas e industrializadas de la monarquía austro-húngara; entre 1918 y 1939 se desarrolló como democracia, formando un estado común con los eslovacos, transcarpatianos y ucranianos, y con importantes minorías alemanas y húngaras; durante la Segunda Guerra Mundial fue un protectorado alemán separado, ocupado por las tropas alemanas. Después de un breve periodo de restauración democrática (1945-1948), el país permaneció bajo la influencia de la Unión Soviética y bajo el régimen comunista hasta la llamada Revolución de Terciopelo en noviembre de 1989. Desde ese momento, se retomó el desarrollo democrático en el país. En 1992, Checoslovaquia se dividió sin problemas en dos

324 nuevos estados democráticos, la República Checa y Eslovaquia. 
Los cambios políticos, económicos y sociales en la antigua Checoslovaquia después de la Revolución de Terciopelo de 1989 atendieron de manera especial a la protección y gestión del medio ambiente. Entre otras circunstancias, se aprobó una nueva legislación relativa al medio ambiente, seguida de cambios en la Administración Pública, efectuados de acuerdo con los principios democráticos clásicos. La protección del medio ambiente encontró un fuerte apoyo ciudadano. En una encuesta realizada en febrero de 1990, los encuestados situaron el medio ambiente sano como la segunda cuestión más importante en sus vidas, después de la libertad (Comité Federal para el Medio Ambiente 1992). Como lógica consecuencia, a principios de la década de los noventa se creó el Ministerio de Medio Ambiente de la República Checa, así como la Agencia para la Conservación de la Naturaleza y protección del Paisaje de la República Checa, a efectos de ejecución de la política pública en materia de conservación de la naturaleza y protección del paisaje, incluidas amplias zonas de protección especial, i.e., en parques nacionales y zonas paisajísticas protegidas.

A estos cambios siguió un rápido desarrollo de la sociedad civil, en el cual las ONG desempeñaron un papel importante.

Este artículo tiene por objeto exponer de manera sintética algunos de los elementos clave del reciente desarrollo, la situación actual y algunas perspectivas de futuro, en materia de naturaleza y de conservación de la biodiversidad. La definición de diversidad biológica (biodiversidad) empleada en este artículo se ofrece en el Anexo 1.

\section{HISTORIA: UNA LARGA TRADICIÓN DE CONSERVACIÓN DE LA NATURALEZA}

Como se muestra en el Anexo 2, el territorio de la República Checa se sitúa en el centro del desarrollo de la biodiversidad en Europa. Los montes Hercinianos en el norte, oeste y sur del país, los montes Cárpatos en el este, las tierras bajas cálidas con hábitats esteparios en el centro, y las zonas panónicas en el sureste, han dado lugar a una diversidad relativamente alta y a una rica naturaleza. Las áreas más significativas son las amplias zonas de bosques de diversos tipos, seguidas de tramos de hábitats esteparios, la tundra boreal, humedales considerables, etc. El territorio ha sido poblado y explotado de manera significativa por diversas oleadas de pobladores, comenzando con la invasión eslava en el s. VI. La fuerte industrialización, especialmente en el norte y oeste, condujo a una importante deforestación. Importante asimismo y con frecuencia con efectos devastadores es la explotación de las minas de carbón en el noroeste de Bohemia (después, en el s. XX, parte del tristemente famoso Triángulo Negro) y norte de la región de Moravia-Silesia. Sin embargo, la explotación humana del paisaje no ha ocasionado exclusivamente problemas medioambientales de importancia, sino que ha dado asimismo lugar al desarrollo de hábitats de gran valor y riqueza de especies, como por ejemplo los prados de siega en Bílé Karpaty (Cárpatos Blancos), con una extraordinaria riqueza de especies vegeta- 
les (una de las praderas de orquídeas más abundante en Europa), o el sistema medieval seminatural de estanques de la zona de Trebon, zonas de humedales de gran importancia, ecológicamente sanas y con gran riqueza de fauna. Las zonas boscosas se han conservado mejor en las montañas. Por decisión de sus propietarios, se reservaron algunas zonas de los bosques vírgenes, y como consecuencia de ello se creó, ya en la mitad del s. XIX, una de las reservas forestales más antiguas de Europa (como el bosque Žofín y Hojná Voda en 1838, el bosque Boubín en 1858, ambos situados en el sur de Bohemia). Puede decirse que los orígenes de la conservación de la naturaleza estuvieron motivados por ideas más o menos románticas (dejar a nuestros hijos "bellas porciones de naturaleza" o un sentimiento de conservación del "patrimonio”) más que por política alguna. Esta particular y original motivación se mantuvo, con algunas excepciones, como principio rector hasta la revolución industrial y el desarrollo de la primera mitad del s. XX, con sus primeros elementos de intensificación. La "cantidad de naturaleza" se siente como enorme, ciertamente suficiente, y por tanto, no había gran necesidad de conservación de aquélla. En lugar de ello, el principio predominante era la necesidad de conocimiento de las especies. En consecuencia, el número de áreas protegidas ha sido bajo durante mucho tiempo, menor del $1 \%$ del total del territorio. Sólo después del intenso desarrollo industrial, en los años treinta, aparecieron los primeros intentos de sistematización de la conservación de la naturaleza. Se crearon decenas de reservas, y por primera vez, pareció surgir el primer principio general de conservación de la naturaleza: cuanto más dañada resulta la naturaleza, menos naturaleza "original" queda en la zona, y más enérgica es la demanda de su protección. En otras palabras, en los tiempos en que el sentimiento de la sociedad era que la naturaleza es suficiente regalo, la conservación no se sentía como algo importante, excepto para la protección de lo extraordinariamente bello. Este principio ha sido aplicado desde entonces hasta la actualidad, por lo que resulta muy difícil proponer la protección preventiva o la conservación de los elementos de la naturaleza que todavía son abundantes. Este fenómeno es de ámbito paneuropeo y puede encontrarse detrás de muchos problemas de establecimiento de la Red Ecológica Europea Natura 2000 en los nuevos Estados miembros de la Unión Europea. Y viceversa, en áreas con daños, destrucción y alteración significativa de la naturaleza, incluso las zonas que no se habrían calificado de muy importantes en condiciones normales, se consideran candidatas para la conservación de la naturaleza. Lógicamente, ello redunda con frecuencia en la protección de zonas menos valiosas que si tal protección se hubiese propuesto en el momento adecuado, antes de la degradación.

El régimen comunista comenzó con una política muy progresista en materia de conservación de la naturaleza. La primera ley sobre protección de la naturaleza en Checoslovaquia, aprobada en 1956, ha sido una de las más modernas y más progresivas en el mundo en materia de protección de la naturaleza. Por desgracia, su gran amplitud no trascendió del papel, aunque al menos, se efectuaron numerosas declaraciones de nuevas reservas naturales, así como de los primeros parques nacionales. Pero la conservación de la naturaleza nunca se aceptó como freno al desarrollo indus326 trial, que se observaba como el principal factor de progreso. Más que valor por sí 
misma, la naturaleza se consideraba como un lugar de esparcimiento para la clase obrera o, en el mejor de los casos, como un elemento de orgullo nacional. El problema más importante, común a todos los entonces países comunistas, y que persiste en la mente de muchos todavía hoy, concernía a la aplicación de la ley y a su cumplimiento. La lucha contra el régimen, desobedeciendo o incluso incumpliendo intencionadamente la ley, se hacía más "normal” con el tiempo, a medida que se prolongaba el régimen comunista, que gobernaba el país con una política muy restrictiva hacia el ciudadano. La cultura del no-cumplimiento y del no respeto a la política estatal se desarrolló con fuerza, aunque principalmente en relación a otras leyes distintas de las relativas a la conservación de la naturaleza. Todo el mundo tuvo oportunidad de observar docenas de casos de falta de cumplimiento de la ley, sin respuesta seria alguna por parte de la Administración, o incluso con persecución de aquellos que querían detener las actividades perjudiciales. Esto dio lugar a un incremento del contacto entre diversos grupos ilegales de ciudadanos, anti-régimen y pro-medio ambiente (incluyendo pro-naturaleza). Sólo a la salida de este periodo tendrá lugar un fuerte apoyo a la nueva política medioambiental, en un ambiente único, con alta aceptación de una enérgica ley de conservación de la naturaleza, que permitirán un desarrollo importante a aquel respecto tras la Revolución de Terciopelo de 1989.

\section{LA LEGISLACIÓN COMO PIEDRA ANGULAR DE UNA CONSERVACIÓN DE LA NATURALEZA Y PROTECCIÓN DEL PAISAJE EFICACES TRAS LA REVOLUCIÓN DE TERCIOPELO}

El avance en la conservación de la naturaleza tras la Revolución de Terciopelo fue extraordinario y sorprendente. En una década aproximadamente, se aprobaron nuevas leyes de carácter muy progresista. La red de Zonas Especialmente Protegidas aumentó con la declaración de nuevas reservas y de diversas zonas protegidas de gran tamaño: entre otros, se declararon tres nuevos parques nacionales, que se unieron al ya existente. Se crearon muchas y diversas asociaciones dirigidas a la conservación de la naturaleza; el número de las llamadas "ONG verdes" se disparó, y su participación en la toma de decisiones aumentó de manera espectacular. Tras unos primeros años de amplia aceptación y apoyo, surgieron los primeros conflictos. Éstos se relacionaron con la perspectiva del desarrollo económico rápido del país. Nuevo ambiente y nueva legislación, comenzando asimismo a advertirse la presencia de las asociaciones ambientales en numerosos conflictos locales, e incluso, aunque más rarameente, en conflictos de ámbito nacional, casi siempre relacionados con la construcción de nuevas infraestructuras, industrias, con la intensificación de la agricultura, etc. Este efecto boomerang existe todavía hoy día y complica el desarrollo de la política de conservación de la naturaleza. No obstante, el sistema se ha basado en unas pocas leyes y decretos (modificados en diversas ocasiones), aprobados originariamente en un "gran boom medioambiental”, tras la Revolución de Terciopelo. Estas leyes y decretos son asimismo los principales elementos o instrumentos de la política actual de conservación de la biodiversidad en la República Checa. 
El principal instrumento legal en la conservación de la naturaleza y protección del paisaje en el país es la Ley del Consejo Nacional Checo N. 114/1992 sobre Protección de la Naturaleza y del Paisaje, con las modificaciones efectuadas posteriormente. La Ley se basa en el enfoque según el cual no sólo deben conservarse para el futuro las zonas especialmente protegidas de la naturaleza, sino que asimismo es extremadamente importante mantener los procesos naturales básicos en el paisaje, haciendo hincapié tanto en la diversidad como en el funcionamiento de los distintos sistemas biológicos. Por tanto, la Ley se basa en un enfoque equilibrado y relativamente moderno, que pone el énfasis en la integridad ecológica, es decir, tanto la diversidad como la importancia del mantenimiento en vida de los procesos de los diversos sistemas biológicos. Además, refleja asimismo las nuevas relaciones propietarias, existentes desde noviembre de 1989. Esta normativa se elaboró no sólo al objeto de recoger los planteamientos actuales en materia de conservación de la naturaleza, protección del paisaje y gestión de la biodiversidad, sino también para cumplir con las obligaciones surgidas de tratados y acuerdos internacionales multilaterales relativos al medio ambiente y a la biodiversidad.

El objetivo de la Ley es contribuir a la conservación y restauración del equilibrio natural en el paisaje, proteger la diversidad de todas las formas de vida, valores y bellezas naturales, así como gestionar de manera sostenible los recursos naturales. Incluye los modos de protección de especies de la fauna y flora (individualmente o por poblaciones enteras), así como de protección y gestión de sus hábitats. Asimismo, se señala en el instrumento legislativo que la protección de los hábitats y la gestión ecológicamente racional de los ecosistemas es el enfoque más rentable a efectos de conservación de la diversidad de especies en un territorio dado.

En virtud de la Ley, todos los animales y plantas silvestres se protegen, por lo general, en todas sus etapas de desarrollo, a excepción de las especies con relevancia desde el punto de vista económico, así como aquellas asociadas a enfermedades (plagas). Se presta especial atención a las especies no autóctonas, puesto que las especies exóticas invasoras, que amenazan los ecosistemas, hábitats y a otras especies, se consideran uno de los riesgos más importantes para la diversidad biológica, incluso en Europa central. Además, todas las especies de aves silvestres cuentan hoy día con un régimen especial de protección, como consecuencia de la transposición de la directiva europea de aves (véase infra).

Los Decretos N.³95/1992 y N. ${ }^{\circ} 175 / 2006$ del Ministerio de Medio Ambiente de la República Checa tienen por objeto la aplicación de la ley. Incluyen, entre otras cuestiones, la lista de especies de fauna y flora silvestres protegidas con carácter especial. Mientras que el primero de aquéllos relaciona 527 especies (o subespecies) como especialmente protegidas, en tres categorías, de acuerdo con el grado de amenaza (46 de hongos, 436 de plantas vasculares, 147 de invertebrados, 17 ictícolas, 28 de anfibios/reptiles, 115 de aves y 38 de mamíferos), el segundo, en particular, transpone los anexos de la normativa europea de conservación de la naturaleza, concretamente 328 de la directiva de aves (Directiva 79/409/CEE relativa a la conservación de aves silves- 
tres) y de la Directiva de hábitats, (Directiva 92/43/CEE del Consejo relativa a la conservación de los hábitats naturales y de la fauna y flora silvestres).

La Ley del Consejo Nacional Checo N. ${ }^{\circ}$ 100/2004 sobre comercio de especies de fauna y flora silvestres en peligro (CITIES) fija las condiciones de importación y exportación de aquellas especies, de conformidad al mismo tiempo con la correspondiente normativa europea, en concreto el Reglamento 338/1994 del Consejo, relativo a la protección de especies de fauna y flora silvestres mediante el control de su comercio.

\section{INCORPORACIÓN A LA UNIÓN EUROPEA, NATURA 2000 Y TENDENCIAS ACTUALES EN MATERIA DE CONSERVACIÓN DE LA NATURALEZA}

La preparación de la República Checa para la incorporación a la Unión Europea, y finalmente su incorporación a aquélla, ha jugado un papel decisivo en el desarrollo de la legislación nacional. En general, ha tenido un impacto positivo importante en el marco legal. Puede decirse, sin lugar a duda, que no se habría alcanzado el nivel actual en materia de conservación de la naturaleza y protección del paisaje si no hubiese existido obligación de poner en práctica la normativa europea. De la misma forma, puede afirmarse que el proceso de incorporación de la normativa europea dio lugar a diversas dificultades y a efectos negativos. La mayoría de ellos resultaron del hecho de que, a diferencia de los antiguos, los nuevos Estados miembros de la Unión (10 países que se incorporaron en 2004, más Rumanía y Bulgaria) tuvieron mucho menos tiempo para aquella transposición y puesta en práctica. Se exigía que los procesos de incorporación, que habían durado en algunos de los 15 Estados Miembros una década o incluso más (por ejemplo, la incorporación de la directiva de aves), finalizasen en el plazo de cuatro a cinco años o incluso en un plazo menor. Al iniciar la enorme cantidad de trabajo a realizar, concurrieron, entre otros factores, la falta de información y experiencia. Además, desde los Estados miembros de la Unión Europea llegaron al país diversos estímulos negativos -se "advirtió" a agricultores, silvicultores, y otros grupos, de que la Red Natura 2000 operaba completamente contra sus intereses. Incluso basándose parcialmente estas "advertencias" en meras cuestiones de detalle o en claros malentendidos en el seno del proceso, crearon una espiral negativa que se añadió a la polémica entre las autoridades administrativas encargadas de la transposición y puesta en práctica y el resto de la sociedad. Ello unido a la cultura heredada del incumplimiento, a los malos recuerdos del régimen administrativo comunista, restrictivo e injusto, y a la durante mucho tiempo esperada oportunidad de desarrollo rápido y de "mejor vida" económica, redundó en serias dificultades en la puesta en práctica de la Red Natura 2000 en la República Checa. Estos mismos efectos, o similares, pueden observarse también en otros nuevos Estados Miembros (UE-12).

El método de puesta en práctica de la Red Natura 2000 en la República Checa ha sido expuesto con detalle en otro lugar. Sus elementos principales se basaron en la normativa y jurisprudencia comunitaria sobre biodiversidad. El Ministerio de Medio Ambiente de la República Checa decidió elaborar en primer lugar, y en cuatro años, un mapa completo de todo el territorio del país. Basándose en los mapas digitalizados 
con información detallada actualizada del estado actual, se propusieron ciertos lugares representativos para su inclusión en la Red Natura 2000, empleando asimismo tanto como fuera posible la red nacional ya existente de Zonas Especialmente Protegidas. El método descrito dio lugar a propuestas de alta calidad y ya evidenciadas. No obstante, requirió grandes recursos de personal: casi 600 botánicos checos participaron en la elaboración del mapa sobre el terreno; numerosos expertos contribuyeron de manera importante al análisis ulterior y más profundo, incluida la gestión y tratamiento de los datos y el cuidadoso examen de las propuestas. La significativa falta de tiempo generó problemas en lo que a presentación pública de propuestas y negociaciones con los propietarios de los terrenos se refiere. A pesar de que se diseñó un sistema integral a estos efectos (alrededor de 120 personas responsables de partes específicas del territorio de la República Checa, más de 300 reuniones y sobre 6000 puestas en contacto con ciudadanos, municipios, industrias y grupos de interés, en sólo ocho meses aproximadamente), el Ministerio se enfrentó a críticas relativas a la insuficiente comunicación con los interesados. La presión desde Bruselas condujo finalmente a la adopción de la propuesta, que aumentó las opiniones negativas, en al menos algunos grupos de interés, sobre cuestiones relativas a la conservación de la naturaleza. La situación actual puede caracterizarse por la permanencia de la polarización de la sociedad. En general, y excepto los sectores verdes de aquélla, la conservación de la naturaleza encuentra más apoyo en la población joven y urbana, en tanto que los habitantes de las zonas más apartadas, que generalmente se hallan en contacto directo con los trabajos de conservación de la naturaleza, toman a menudo posiciones en sentido negativo o en el mejor caso neutral. Las tensiones continúan también en los casos de desarrollo de infraestructuras y de proyectos urbanísticos locales (por ejemplo los de gran escala y de turismo de masas, etc.). Se han llevado a cabo muchos intentos, algunos de ellos con éxito, de flexibilizar la legislación a través de modificaciones ad hoc en el Parlamento nacional. Pueden observarse asimismo otras tendencias. En general, ha aumentado la cantidad (cobertura) de Zonas Especialmente Protegidas. El déficit financiero también está aumentando, en particular en relación con la gestión de las Zonas Especialmente Protegidas, aunque esta tendencia se encuentra hoy día ligeramente aminorada gracias a los Fondos Estructurales europeos.

Además, en general, los que se presentan visiblemente como grandes problemas del medio ambiente se resuelven decididamente paso a paso, como por ejemplo la calidad del aire y del agua que ya han mejorado algo. Como consecuencia, el sentimiento de premura en la conservación de la biodiversidad ha descendido. Por regla general, se ha declarado públicamente el apoyo a la conservación de la naturaleza (Comisión Europea 2008), pero la actitud cambia sensiblemente en relación a propuestas específicas (el efecto NIMBY). En zonas y especies "comunes" o "no protegidas" se amplifican las tendencias negativas. Si bien la política se pone en práctica de manera activa y se cumple, ello a menudo conduce a respuestas negativas. La única excepción se encuentra vinculada a los instrumentos financieros -existe una actitud positiva hacia el apoyo financiero a la gestión, como por ejemplo a los programas agroambientales. Sin embargo, muy a menudo se contemplan más como apoyo fi330 nanciero a los agricultores que como medidas de apoyo a la biodiversidad. 
El objetivo comunitario de detener la pérdida de biodiversidad antes de 2010 ha tenido muy poco o ningún efecto en el público en general, en parte porque los objetivos de carácter nacional se consideran más importantes que los de carácter europeo, y en parte por la insuficiencia de información. El público sólo se interesa por cuestiones relativas a la conservación de la naturaleza y protección del paisaje relacionadas con controversias muy ostensibles, a menudo politizadas, como por ejemplo la polémica relativa a la no intervención en los bosques en parques nacionales. Parece que el reto de la próxima etapa será motivar nuevamente al público a efectos de lograr un acercamiento activo a la conservación de la naturaleza, e incrementar en ciertos grupos de ciudadanos (los grupos clave) la aceptación de la política de conservación de la naturaleza.

\section{ELEMENTOS PRINCIPALES DE LA POLÍTICA ACTUAL DE CONSERVACIÓN DE LA NATURALEZA}

Sobre la base de la complicada historia y de las diversas influencias recibidas, según se ha expuesto más arriba, la política de conservación de la naturaleza en la República Checa ha ido evolucionando. El marco europeo aún no se ha puesto en práctica en su totalidad, y el sistema nacional requiere de estabilización y actualización, incluso si en su estado actual es, en principio, funcional y basado en la ciencia. Los problemas clave a los que hay que enfrentarse son parecidos a los de cualquier política medioambiental: (a) multidimensionalidad (la diversidad de resultados significa que toda política genera ganadores y perdedores); (b) incertidumbre científica (incluso si intentamos diseñar una política de conservación de la naturaleza basada en enfoques científicos, seguirá existiendo un amplio campo de ignorancia e inseguridad, debido a la falta de conocimiento); (c) conflictos de valores (por ejemplo, el conflicto clásico entre el valor económico real, o monetario incluso, de otros bienes y servicios en la economía, comparado con el valor patrimonial o valor intrínseco de la naturaleza y el paisaje); (d) desconfianza (como resultado de los factores de controversia mencionados arriba) y (e) urgencia (dado el rápido descenso y pérdida de biodiversidad, en todos sus niveles principales). Toda política debe tratar estos problemas, que se encuentran también en relación con el apoyo, reducido y cambiante, del público en general. La situación en muchos países sugiere que sólo puede conseguirse una solución adecuada e integral a través de un método paso a paso. La exposición de carácter general que se realiza a continuación muestra algunos de los elementos de la actual política de gestión en materia de conservación de la naturaleza, protección del paisaje y biodiversidad en la República Checa.

\section{Búsqueda de prioridades estratégicas}

Como en otros lugares, debido a la falta de tiempo y de fondos, es preciso establecer prioridades estratégicas en materia de conservación y gestión de la biodiversi- 
dad, así como en materia de empleo sostenible de sus elementos componentes. La planificación en materia de conservación, basada en la mejor ciencia disponible, intenta dar respuesta a la pregunta: ¿qué proteger primero? Las decisiones finales se hallan en relación con el marco político, social y económico en el cual ha de operar la conservación de la naturaleza y la gestión de la biodiversidad (Margules \& Sarkar 2007).

Desde 1998, ha sido el Gobierno el que ha elaborado los principales documentos de índole política que afrontan la cuestión de la conservación de la naturaleza, protección del paisaje y gestión de la biodiversidad. Entre aquéllos se encuentra los informes Política Ambiental Estatal de la República Checa 2004-2010 (ministerio de medio ambiente de la república checa 2004); Estrategia de la República Checa para el DesarroIlo Sostenible (oficina del gobierno de la república checa 2004a); Estado de Conservación de la Naturaleza y Programa de Conservación del Paisaje (Mlčoch et al., 1998) aprobado por el Gobierno en 1998, que actualmente se encuentra el proceso de actualización y, especialmente, el informe general Estrategia Nacional de la República Checa en materia de biodiversidad (Ministerio de medio Ambiente de la República Checa 2005) 3 .

\section{1. Áreas protegidas (AAPP)}

La tan debatida cuestión SLOSS (una única y amplia reserva o diversidad de reservas de reducido tamaño, Shafer, 1995; Schwartz, 1999; Groom et al., 2005) puede tratarse de manera efectiva a través de la delimitación de varias zonas de protección con diferentes normas de conservación y gestión, dentro de las amplias áreas especialmente protegidas. En la República Checa, la vigente legislación nacional combina ambos planteamientos, con un total de 4 parques nacionales, 25 áreas paisajísticas protegidas y un conjunto de al menos 2.000 áreas protegidas de menor tamaño, de diferentes categorías (Reservas Nacionales, Monumentos Naturales Nacionales, Reservas Naturales y Monumentos Naturales). En la actualidad, todos los lugares propuestos para su inclusión en la Red Natura 2000 se deben incorporar a alguna de las categorías existentes de Zonas Especialmente Protegidas, en el plazo de seis años tras su inclusión en la lista europea. No obstante, la próxima modificación de la ley de protección de la naturaleza propondrá también la llamada "protección básica" para aquellas áreas de la Red Natura 2000 en las que los regímenes de protección existentes se revelan excesivos. En lo que concierne a la Red Natura 2000, la República Checa cuenta actualmente con 39 Zonas de Protección Especial (ZEPA) en cumplimiento de la directiva de aves, y al menos un centenar de Lugares de Importancia Comunitaria (LIC).

3 Las referencias documentales realizadas en el presente artículo se encuentran traducidas a lo largo del cuerpo del mismo, con vistas a la mejor comprensión de aquél, si bien en la bibliografía se han mantenido en el idioma original, para mayor facilidad en su localización (nota del traductor). 


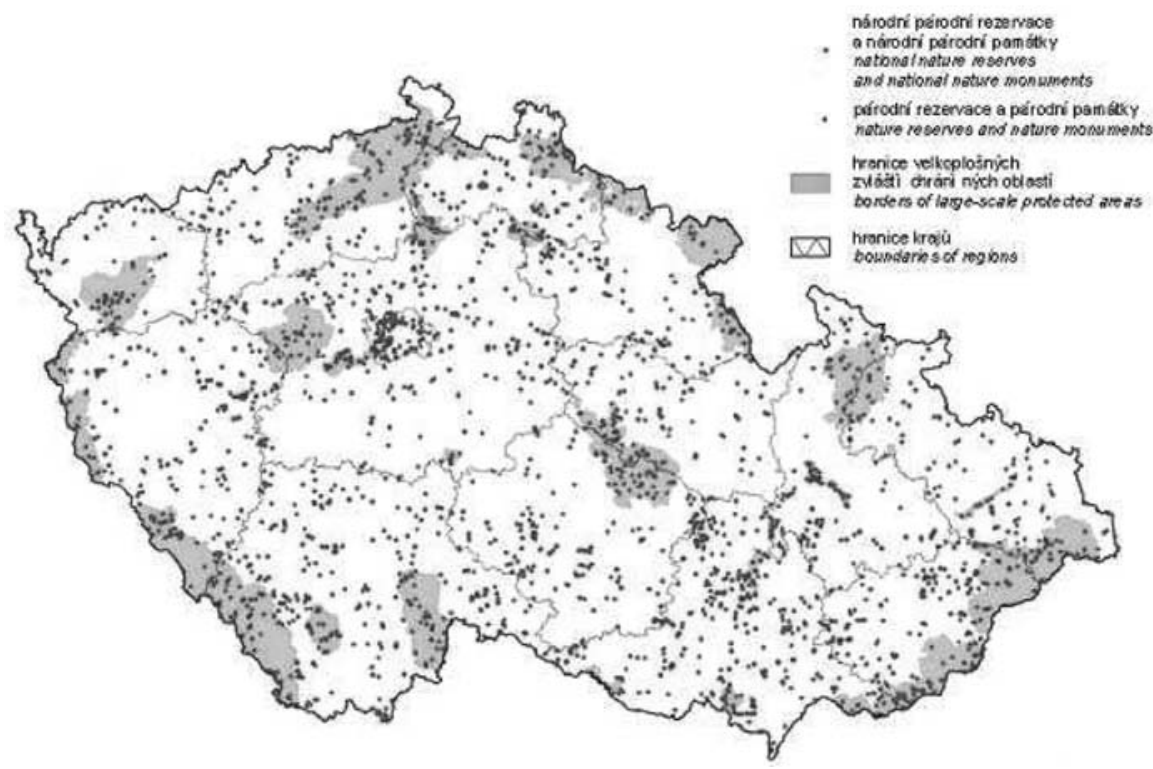

Fuente: Agencia para la Conservación de la Naturaleza y Protección del Paisaje de la República Checa.

\section{FIGURA 2}

Áreas Protegidas de gran tamaño en la República Checa a fecha 1 de enero de 2009

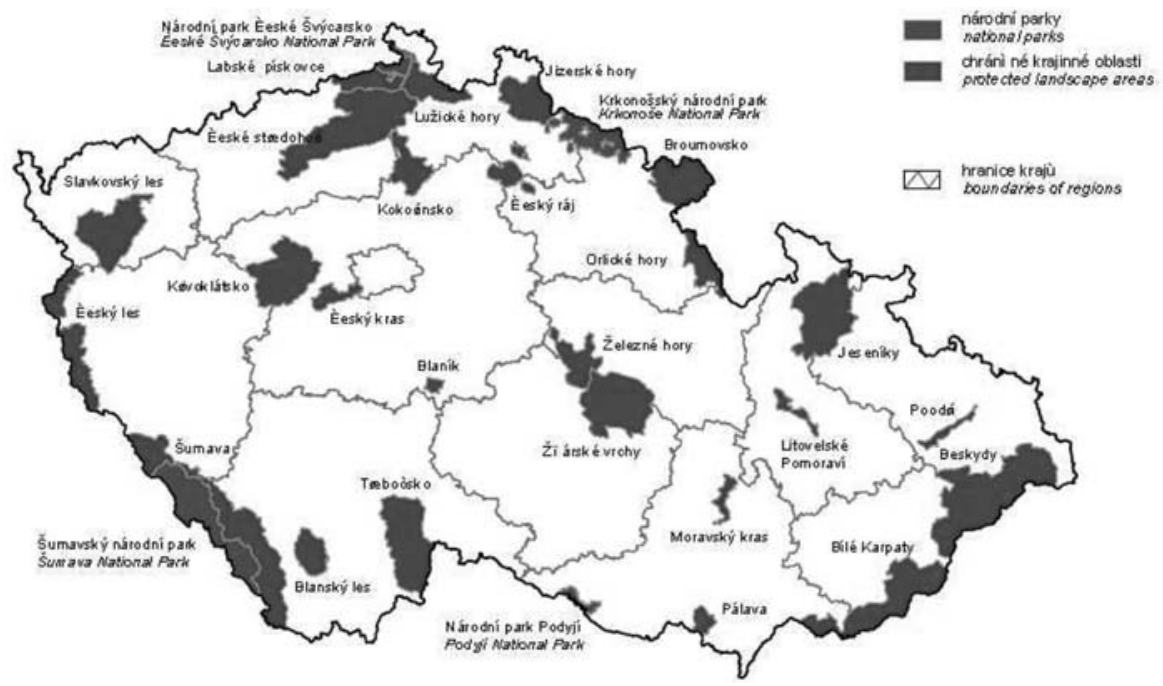

Fuente: Agencia para la Conservación de la Naturaleza y Protección del Paisaje de la República Checa. 


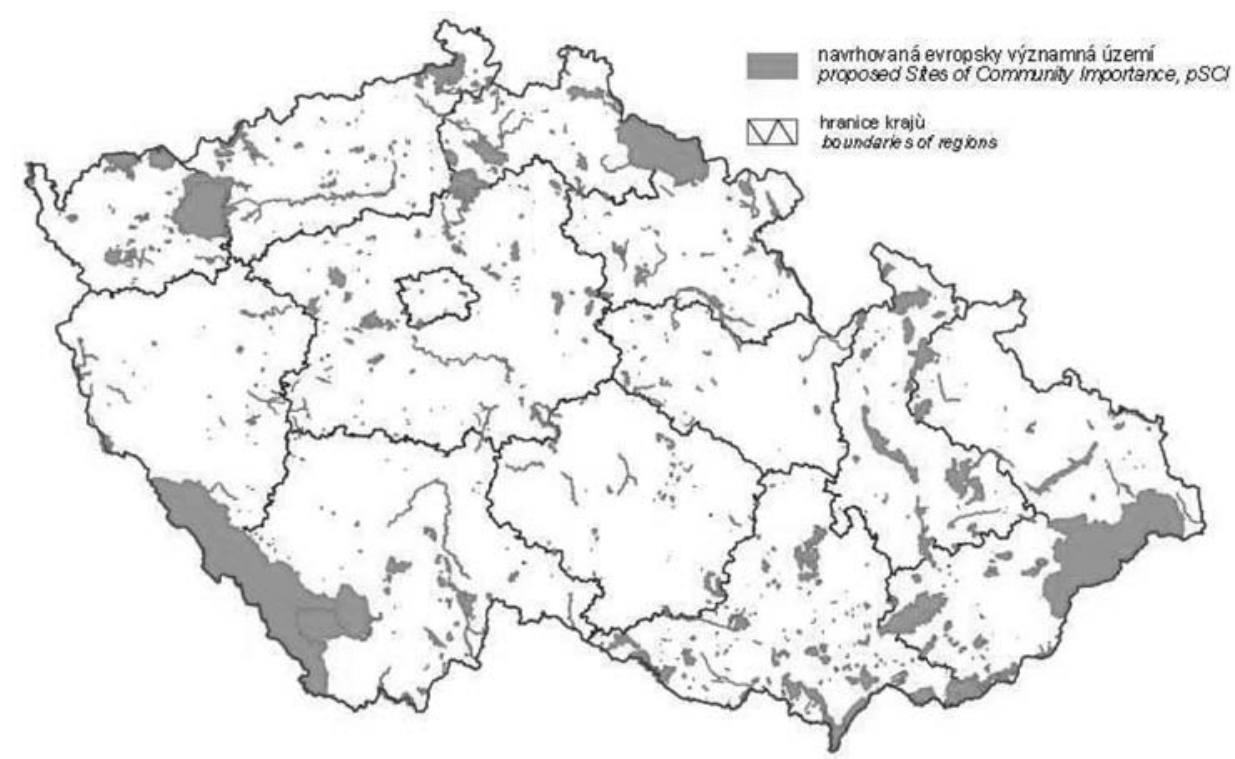

Fuente: Agencia para la Conservación de la Naturaleza y Protección del Paisaje de la República Checa.

\section{Paisajes no reservados y protección del paisaje}

Además de las Zonas Especialmente Protegidas y de la protección de las especies silvestres en peligro, se protege en general en toda la República Checa el paisaje existente globalmente considerado, y en particular una selección de algunos de sus elementos componentes. En cumplimiento de la más arriba referida Ley de conservación de la naturaleza, los instrumentos de protección general se centran en el paisaje no incluido en las áreas protegidas (el paisaje no protegido) e incluyen (1) parques naturales, (2) Sistemas Territoriales de Estabilidad Ecológica del Paisaje (TSES), una red ecológica a varios niveles (local, infra-nacional y nacional), (3) elementos de importancia del paisaje, (4) entorno del paisaje, (5) árboles memoriales/centenarios, (6) especies de árboles no forestales, (7) parcelas protegidas temporalmente, (8) elementos naturales abióticos como cuevas y otros fenómenos kársticos.

A pesar de algunos defectos y de problemas aún no resueltos, especialmente 334 en lo que al auténtico papel de los pasillos biológicos/ecológicos (biocorredores) se 
refiere, a efectos de posibilitar el movimiento de los organismos a lo largo del paisaje (Hilty et al., 2006; Boitani et al., 2007; Corlatti et al., 2009), el TSES es un instrumento importante para las autoridades estatales en materia de conservación de la naturaleza en lo que respecta al acceso a la naturaleza en el paisaje no protegido. La República Checa se encuentra entre los países europeos que han incluido en la legislación de conservación de la naturaleza y gestión del paisaje la creación y gestión de redes ecológicas a diversas escalas territoriales. Además, la materia se ha convertido asimismo en una parte de la legislación relativa a la planificación territorial del país, i.e. la Ley N. ${ }^{\circ} 183 / 2006$ sobre planificación urbana y rural y el Código de Edificación (la Ley de Edificación). Por tanto, el sistema TSES ha adquirido un carácter obligatorio general en el seno del proceso de aprobación de la documentación para la ordenación territorial. En la práctica, la red ecológica debe asimismo tomarse en consideración en la elaboración de las propuestas de reparcelación integral del terreno, y del Plan de Gestión Forestal (instrumentos básicos de gestión del uso forestal por los propietarios, tanto privados como públicos). Los encargados de elaborar la planificación territorial desean, por lo general, destinar diversos terrenos a la conservación de la naturaleza y protección del paisaje, pero, especialmente a escala local, la identificación de los elementos componentes de la TSES realizada por los municipios, en tanto autoridades estatales de conservación de la naturaleza, es generalmente de baja calidad. Además, la propia red ecológica se considera con mucha frecuencia exclusivamente como simple trámite burocrático (Mackovčin, 2000; Mackovčin et al., 2005, Plesník, 2008).

La teledetección, herramienta utilizada para la elaboración de los mapas CORINE de coberturas del suelo, ha revelado que la República Checa es una de las partes de Europa con el mayor índice de expansión urbana descontrolada. La colaboración con los encargados de elaborar la planificación territorial se revela decisiva para el futuro de la naturaleza y el paisaje en la República Checa.

La República Checa fue el primer país poscomunista en el cual el Ministerio de Medio Ambiente contó con incentivos fiscales para los propietarios a efectos de gestión racional desde el punto de vista ecológico, incluso fuera de las áreas protegidas. Aquéllos incluyen, entre otros, los programas de gestión del paisaje del Ministerio de Medio Ambiente (Programa de Gestión del Paisaje, Programa de Restauración del Sistema Fluvial). Su objetivo consiste en apoyar las medidas de protección del status cultural del paisaje, con lo que contribuyen a mantener la diversidad de especies, a mejorar los procesos naturales en el paisaje, a fortalecer las funciones de los principales elementos componentes de aquél y del sistema de estabilidad ecológica, etc. Es decisivo el hecho de que la gente no ha considerado a las autoridades estatales en materia de conservación de la naturaleza desde una perspectiva limitada, como aquéllos que imponen restricciones, sino también como la autoridad de puesta en práctica de los intereses públicos, a través de incentivos razonables. 


\section{FIGURA 4}

Sistema Territorial de Estabilidad Ecológica del Paisaje (TESES - niveles supra-regional y regional) en la República Checa a fecha 1 de enero de 2009

\section{SUPRAREGIONAL AND REGIONAL TSES OF THE CZECH REPUBLIC}

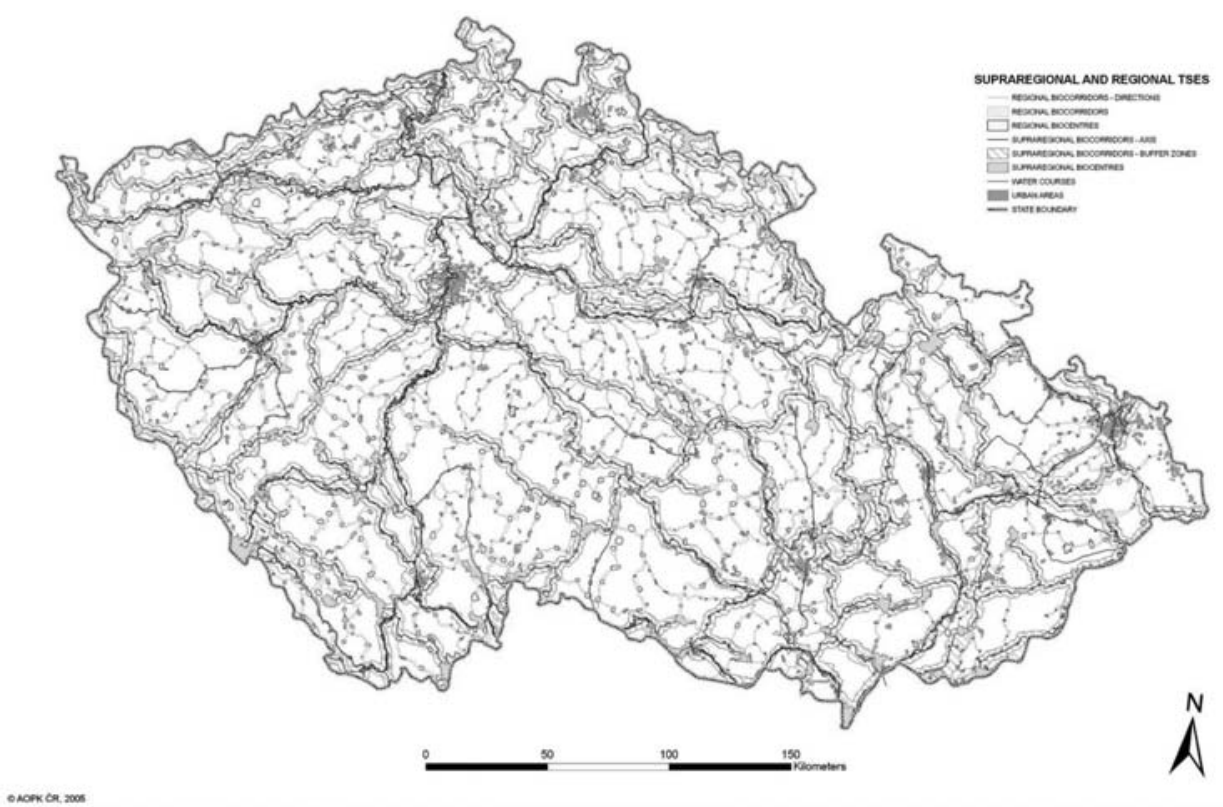

Fuente: Agencia para la Conservación de la Naturaleza y Protección del Paisaje de la República Checa.

\section{Enfoque participativo}

Dados los 40 años de sistema jurídico comunista, de carácter restrictivo, el enfoque participativo no ha sido casi nunca puesto en práctica en el pasado; hoy día se está introduciendo, poco a poco, en el sistema de conservación de la naturaleza, protección del paisaje y gestión de la biodiversidad. No obstante, queda mucho por hacer.

La normativa vigente en materia de conservación de la naturaleza y protección del paisaje en la República Checa puede caracterizarse de manera general por el uso de instrumentos de naturaleza administrativa, pudiendo adoptarse desde decisiones de carácter vinculante por parte de las autoridades estatales en materia de conservación de la naturaleza, a prohibiciones de ciertas actividades, o a excepciones a aquéllas. La parte fundamental de la normativa, que establece derechos y deberes en materia de conservación de la naturaleza y protección del paisaje, es la más arriba referida Ley de Protección de la Naturaleza y del Paisaje, con las modifi- 
caciones que ha sufrido, Ley que incluye entre otras una disposición especial, de acuerdo con la cual las zonas o lugares con valor natural, o incluso los árboles, pueden protegerse por contrato. Los esfuerzos en orden al empleo del recurso contractual en la conservación de la naturaleza se refieren especialmente a los Lugares de Importancia Europea (referidos en la Ley con la expresión Lugares de Importancia Comunitaria, LIC, de conformidad con la Directiva de Hábitats), que son parte de la red europea Natura 2000. En lo que a los LIC se refiere, la Ley permite que la conservación pueda llevarse a cabo por contrato, en lugar de estableciendo una Zona Especialmente Protegida. De este modo, los propietarios o terratenientes pueden negociar las condiciones de conservación, en lugar de imponerles las condiciones establecidas directamente por la Ley para la Zona Especialmente Protegida en cuestión. No obstante, estos contratos han dado lugar a algunas complicaciones jurídicas, obstáculos y problemas.

La modificación de la Ley de protección de la Naturaleza y del Paisaje que tuvo lugar en 2004 incrementó los requerimientos de compensación financiera por parte de los propietarios de terrenos agrícolas, bosques y estanques de piscicultura, puesto que la producción se hizo más difícil debido a las restricciones impuestas por la referida Ley o a las decisiones tomadas por los órganos estatales de conservación de la naturaleza en cuanto autoridades administrativas estatales.

A diferencia de algunos países de Europa occidental, en la República Checa los fideicomisos inmobiliarios sólo se han activado a partir de la mitad de los años noventa. En 2008, se han creado 55 fideicomisos inmobiliarios, gestionados por la ONG más importante en materia de conservación de la naturaleza, la Unión Checa para la Conservación de la Naturaleza, en el seno del proyecto Un lugar para la naturaleza. No se ha establecido ninguna zona protegida por persona física.

\section{Interrelación política-ciencia y evaluación de la biodiversidad}

Aún cuando los principios en materia de conservación del estado de la naturaleza se basan en la medida de lo posible en la ciencia, el conocimiento todavía es limitado. Obviamente, esta insuficiencia en el conocimiento científico no debe impedirnos tomar medidas activas, habida cuenta de que el cambio decisivo puede tardar mucho tiempo. Al mismo tiempo, es necesario continuar con el desarrollo de todos los factores de relevancia del enfoque científico (Pielke, 2002; Briggs, 2006). Los elementos principales de este proceso (investigación, seguimiento, indicadores de evaluación de la situación y tendencias, por un lado, y eficacia de la aplicación de la política, por otro, previsiones ecológicas y escenarios de desarrollo, planificación de la conservación, etc.) se han desarrollado y coordinado por la Agencia para la Conservación de la Naturaleza y protección del Paisaje de la República Checa, que es asimismo responsable en particular de informar de las obligaciones existentes de acuerdo con la normativa europea. Entre las obvias limitaciones se encuentra la falta de medios, tanto en términos financieros como en términos de personal y disponibilidad 
del saber hacer y de la mejor praxis. Por otro lado, la elevada calidad actual del trabajo de la Agencia se ha visto recompensada con su participación en el Centro Temático Europeo de Diversidad Biológica, creado por la Agencia Europea de Medio Ambiente de Copenhague. El apoyo financiero a las actividades más relevantes aumentó durante la elaboración de los mapas para la aplicación de la red Natura 2000, y posteriormente a través de la coordinación de la observación y seguimiento de la biodiversidad, proponiéndose entre otras cosas el suministro al escala europea de los datos pertinentes, a efectos de seguimiento e informe (en cumplimiento del artículo 17 de la Directiva de Hábitats).

\section{Información, educación y concienciación pública (CEPA)}

En la República Checa, ha existido una tradición relativamente larga de información, educación y concienciación pública en materia de conservación de la naturaleza, llevada a cabo tanto por el Gobierno como por las ONG.

Las autoridades estatales para la conservación de la naturaleza desarrollan las actividades de información, educación y concienciación pública, tales como técnicas educativas, paneles de información ubicados cerca de fenómenos naturales específicos, documentos explicativos de temporada, etc. Son algunas de las medidas más efectivas a este respecto. No existen todavía, sin embargo, centros para visitantes que expongan de manera atrayente los motivos y argumentos para la conservación de la naturaleza y protección del paisaje en las zonas concretas de que se trate. Por ello, en 2008 la Agencia para la Conservación de la Naturaleza y Protección del Paisaje de la República Checa puso en marcha el nuevo programa $L a$ Casa de la Naturaleza. Para 2015 se prevé construir en las Zonas Especialmente Protegidas más visitadas del país y de mayor valor una red de centros para visitantes, bien equipados y de carácter multifuncional. El programa se ha financiado en su mayor parte a través de los fondos europeos (Programa Operativo de Medio Ambiente), con un total de 700 millones de coronas checas (aproximadamente 26 millones de euros).

A menudo en colaboración con las autoridades locales y regionales, las ONG han creado centros de educación ecológica y medio ambiental, que tienen por destinatarios tanto al público en general como a grupos clave (escolares, jóvenes, profesores, agricultores, cazadores, turistas y visitantes de las Zonas Especialmente Protegidas, etc.).

En el futuro, se emplearán en mayor medida las tecnologías actuales para la información y los instrumentos de comunicación (los "nuevos medios"), tales como Internet, incluidas las redes sociales como Facebook y los blogs, los teléfonos móviles o la distribución de archivos multimedia, con a aumentar la concienciación pública en materia de conservación de la biodiversidad y empleo sostenible de sus elementos 338 componentes, y especialmente para llegar a los jóvenes. 


\section{NATURALEZA Y CAMBIO CLIMÁTICO COMO NUEVO RETO: UNA HISTORIA DE MITIGACIÓN Y ADAPTACIÓN}

Como resultado del cambio climático, la República Checa deberá hacer frente a los impactos desfavorables de aquél en el medio ambiente y en la calidad de vida. En consecuencia, es necesario adoptar medidas en orden a mantener el cambio climático dentro de límites aceptables (medidas de mitigación). En todo caso, son ya inevitables ciertos cambios. Por tanto, como en cualquier otro lugar es importante prestar asimismo atención a las medidas de adaptación, que nos permitan adaptarnos a los cambios del clima en el futuro, por ejemplo aumentando la capacidad de retención de agua del territorio, teniendo en cuenta la frecuencia creciente del fenómeno de las lluvias extremas.

\section{Política nacional de mitigación y adaptación al cambio climático}

En 2004, el Gobierno de la República Checa elaboró el documento titulado Programa Nacional de Mitigación de los Impactos del Cambio Climático en la República Checa (Ministerio de Medio Ambiente de la República Checa, 2004b). Recientemente, se ha preparado un ulterior documento (Política de Protección del Clima en la República Checa (Ministerio de Medio Ambiente de la República Checa, 2009a). Se debe responder con eficacia no sólo a los nuevos conocimientos en la materia, sino también a los avances recientes en la protección del clima en la Unión Europea y a nivel global. El documento tiene por objeto tanto la progresiva reducción de las emisiones de gases de efecto invernadero como la atenuación de los impactos del cambio climático en la República Checa.

La alta diversidad de hábitats, así como la relativamente amplia gama de escenarios en que operará el cambio climático previsto, ha complicado considerablemente la evaluación de los posibles impactos de aquél en la diversidad biológica existente en la República Checa. Además, están previstos en los actuales escenarios el calentamiento, la prolongación del periodo de vegetación, cambios en el régimen estacional de precipitaciones, ausencia de humedad ocasionada por el aumento de la evapotranspiración (la suma de evaporación y transpiración en una zona en concreto) y el aumento de la frecuencia de fenómenos extremos, como los periodos de sequía, tormentas, inundaciones y tornados. Entre los ecosistemas más vulnerables al cambio climático en la República Checa se encuentran los ecosistemas montañosos y los ecosistemas de las principales praderas residuales (Pretel, 2009).

Mientras que la política gubernamental ofrece respuesta de manera especial a la urgente necesidad de estabilizar y reducir las concentraciones de gases de efecto invernadero en la atmósfera, no existe una estrategia integral de adaptación a los cambios climáticos, y por tanto resulta necesario adoptar con carácter nacional un programa de adaptación o medidas estratégicas. La Dirección de Conservación de la 
Naturaleza y Protección del Paisaje del Ministerio de Medio Ambiente de la República Checa ha elaborado un proyecto de estrategia integral en materia de medidas de adaptación del paisaje en relación con el cambio climático, como base de la ya referida política gubernamental (Pelc, 2009). La estrategia no se limita a la simple descripción de los posibles impactos del cambio climático actual y previsto en el paisaje en su conjunto y en sus elementos componentes, sino que también propone medidas específicas para hacer frente de manera razonable a las consecuencias del cambio climático en la naturaleza y en el paisaje en la República Checa. Se basa, entre otros, en el Libro Blanco de la Comisión Europea (Comisión Europea, 2009), elaborado recientemente. Debe decirse que a pesar del enorme desarrollo que han experimentado recientemente los métodos de previsión ecológica, algunas de las estimaciones realizadas pueden dar lugar a cierto nivel de inseguridad.

Los ecosistemas sanos, con buen funcionamiento y con niveles normales de biodiversidad tienen mayor capacidad que los ecosistemas pobres y dañados para resistir y recuperarse más fácilmente de los sucesos meteorológicos extremos (Naeem et al., 2009). Tienen además mayor capacidad para proporcionar servicios del ecosistema al hombre. La restauración razonable de los ecosistemas dañados es asimismo un factor importante para adaptar el paisaje al cambio climático. Respecto a la adaptación al cambio climático a escala de ecosistema y de paisaje, las medidas preventivas pueden ser más efectivas y menos costosas que la inactividad o la acción a posteriori (Stern, 2006).

\section{Adaptación basada en los ecosistemas}

A efectos de la referida puesta en práctica a tiempo de medidas eficaces de adaptación en el paisaje, pueden aplicarse las basadas en los ecosistemas. La adaptación basada en los ecosistemas consiste en el empleo de actividades de gestión sostenible, a efectos de sustentar la adaptación planificada. Entre otras cuestiones, localiza y pone en práctica una serie de estrategias para la gestión, conservación y reparación de los ecosistemas, con objeto de proporcionar servicios que permitan a las personas adaptarse a los impactos del cambio climático. Pretende aumentar la resistencia y capacidad de recuperación y reducir la vulnerabilidad de los ecosistemas y de las personas más afectadas por el cambio climático. Las principales medidas de adaptación incluyen: (a) el aumento de la conectividad (diseñando corredores, eliminando las barreras a la dispersión, creando reservas cercanas unas de otras, reforestando); (b) la integración en la planificación de los impactos del cambio climático (diseño de zonas protegidas); (c) la atenuación de otras amenazas, como por ejemplo las especies exóticas invasoras, fragmentación del hábitat, sobreexplotación de los recursos biológicos (Heller \& Zavaleta 2009, Mawdsley et al., 2009).

La adaptación basada en los ecosistemas se integra adecuadamente en el seno 340 de procesos más amplios de adaptación, pudiendo aplicarse las estrategias de desa- 
rrollo a los niveles paneuropeo, de la Unión Europea, nacional, infra-nacional, y local. No obstante, como todas las medidas de adaptación, las basadas en los ecosistemas no están exentas de complejidad y de riesgo. En consecuencia, es importante que las decisiones de empleo de aquellas medidas se sometan a procedimientos de gestión del riesgo y de coste-eficacia. Una buena planificación y aplicación de medidas de adaptación puede servir asimismo como instrumento económico y muy eficaz a efectos de mitigar/adaptar otros impactos del cambio climático en la sociedad (por ej. inundaciones, olas de calor, etc.). Es casi imposible resolver el problema de la conservación de la naturaleza sin enfrentarse al problema del cambio climático; pero, de igual manera, es imposible resolver el problema del impacto del cambio climático sin abordar la cuestión de la biodiversidad y de los ecosistemas (Miko \& Zaunberger, 2009; Plesník \& Pelc, 2009). Además, a efectos de puesta en práctica de la adaptación basada en los ecosistemas, resulta necesario adoptar un enfoque en la gestión que presente capacidad de adaptación, permitiendo realizar ajustes en aquélla, en respuesta a los cambios en las presiones externas, así como a la incertidumbre existente respecto del funcionamiento del ecosistema.

\section{Gestión adaptativa}

En el pasado, la mayoría de los ecologistas creían que había un "equilibrio de la naturaleza". En consecuencia, se suponía que los ecosistemas se encontraban generalmente en un estrecho equilibrio, de carácter determinista, con los controles medioambientales. Los fundadores del movimiento ecológico creían en la importancia de un pasado biológico y geológico, así como en los patrones naturales y en la coherencia inherente, que permanece a lo largo del tiempo. La piedra angular del paradigma clásico era la teoría de la sucesión climática. Tal fue la idea de la dinámica del ecosistema que dominó hasta principios de la década de los setenta.

A mediados de los noventa, las suposiciones pasadas y los principios de la ecología y de la conservación biológica habían cambiado, con el nuevo paradigma del "noequilibrio", que hace hincapié en el hecho de que los sistemas ecológicos se encuentran abiertos a la influencia externa, son frecuentemente no deterministas y casi nunca se encuentran en estado de equilibrio. La naturaleza debe ser observada como un mosaico que fluye, formado por diversidad de hábitats cambiantes (el "flujo de la naturaleza" -Pickett et al., 1992, 1997; Odum, et al., 1995; Hanski \& Gaggiotti, 2004; Rohde, 2006; Hobbs \& Suding, 2009).

Por tanto, la naturaleza puede conservarse y gestionarse de manera adaptativa, empleando procesos iterativos que conduzcan a decisiones óptimas ante la incertidumbre. La gestión adaptativa es un proceso sistemático para la mejora constante de las políticas y prácticas, aprendiendo de los resultados a que conducen los programas operacionales, y empleando asimismo enfoques quasiexperimentales; el flexible “aprender haciendo" versus la gestión exhaustiva, etc. (Holling, 1978; Walter 1986; Pickett et al., 1997). 


\section{POLÍTICA DE CONSERVACIÓN DE LA NATURALEZA Y RETOS EN LA PRÓXIMA ETAPA: ¿RECUERDOS DEL FUTURO?}

Toda futura política de conservación de la naturaleza deberá ciertamente basarse en el sistema actual, que puede completarse o modificarse como consecuencia de los hechos. La agenda mundial (UNEP, CBD, etc.), paneuropea y de la Unión Europea (las Directivas de Aves y Hábitats, el Plan de Acción europeo para la biodiversidad, etc.) influirá incuestionablemente de manera significativa en este desarrollo. No obstante, deberán asimismo respetarse en el futuro las características especiales existentes como resultado del desarrollo en la República Checa.

Un objetivo fundamental obvio de este proceso es preservar la integridad ecológica y de los ecosistemas y su capacidad de funcionamiento (funcionalidad), asumiendo que la conservación de la naturaleza es cuestión de interés público y que una naturaleza sana significa gente sana, población sana. Una de las herramientas a emplear a tal efecto es la consolidación y codificacion sistemática de la normativa de conservación de la naturaleza. Este proceso debe seguir una serie de normas y principios, a saber:

1. Mantenimiento de poblaciones de especies autóctonas numerosas y por tanto genéticamente viables en el seno de hábitats no contaminados y suficientemente amplios.

2. Mantenimiento de los procesos sobre los que descansa la vida en los ecosistemas y que mantienen estos últimos (y que se conocen con la expresión de servicios del ecosistema).

3. Mantenimiento de una representación adecuada de los tipos de usos del hábitat/ecosistema/terreno, en relación con su carácter naturalmente heterogéneo.

4. Integración de los elementos de conservación de la naturaleza en otras políticas de importancia con influencia en la naturaleza y el campo (agricultura, silvicultura, transporte, energía, planificación territorial, etc.).

5. Diversificación de los enfoques, excluyendo el planteamiento único. Los planteamientos tradicionales en materia de conservación, gestión activa, gestión adaptativa, protección/conservación del paisaje y de la naturaleza no protegida/paisaje no protegido, planteamientos más arriesgados, etc.; todos han de tener su valor en las condiciones específicas de las distintas zonas.

6. Aplicación efectiva y, en caso de incumplimiento, ejecución forzosa eficaz. Comunicación permanente con todos los interesados, recurso a planteamientos "suaves" e instrumentos económicos, puesta en práctica del enfoque participativo: la conservación de la naturaleza para la gente y con la gente.

342 7. Satisfacción del interés humano dentro de los límites referidos. 
A efectos de conservación de la biodiversidad, y gestión y empleo sostenible de sus elementos, resulta asimismo crucial advertir la importancia de los servicios del ecosistema y encontrar la manera de pagar por ellos, de internalizar al menos alguna de las externalidades del sistema económico. En este contexto, la iniciativa de estudio en curso del TEEB (La Economía de los Ecosistemas y la Biodiversidad, comunidades europeas 2008) puede jugar un papel decisivo. Resulta obvio que no todo lo relacionado con la biodiversidad y los ecosistemas ha de tener valor económico. Por otro lado, la economización del debate puede redundar en una compresión más fácil del decisivo papel que desempeña la biodiversidad para el ser humano, en cuanto base de su bienestar y en cuanto seguro de supervivencia a largo plazo. En particular, en las condiciones de desarrollo económico de los países poscomunistas, ello puede tener una gran importancia a efectos de conservación de un status bastante bueno, o por lo menos algunos de los elementos componentes de la naturaleza en aquéllos, antes de que resulte dañado y sea considerado por el público en general como algo verdaderamente "poco común", por ejemplo, antes de que el país alcance el status de alguno de los países de Europa occidental con más influencia del hombre, antes de que sea necesario adoptar medidas intensivas de alto coste, temporal y de recursos humanos.

\section{BIBLIOGRAFÍA}

Boitani L., Falcucci A., Maiorano L. \& Rondinini C. (2007): Ecological networks as conceptual frameworks or operational tools in conservation. Conserv. Biol. 21: 14141422.

Briggs S.V. (2006): Integrating policy and science in natural resources: why so difficult? Ecol. Manage. Rest. 7: 37-39.

Commission of the European Communities (2008): Attitudes of European towards the issue of biodiversity. Flash Eurobarometer Series N. ${ }^{\circ}$ 219. European Commission Brussels, $71 \mathrm{pp}$.

Commission of the European Communities (2009): Adapting to climate change: Towards a European framework for action. White paper. COM (2009) 147 final. Commission of the European Communities Brussels, 17 pp.

Corlatti L., Hacklander K. \& Frey-Roos F. (20069): Ability of wildlife overpasses to provide connectivity and prevent genetic isolation. Conserv. Biol. 23: 548-546.

EEA (2006): Urban sprawl in Europe. The ignored challenge. Office for Official Publications of the European Communities Luxembourg, 56 pp.

European Communities (2008): The economics of ecosystems \& biodiversity. An interim report. European Communities Brussels, 64 pp.

Federal Committee for the Environment (1992): State of the environment in Czechoslovakia. Vesmir Publ. Prague, 120 pp.

Groom M.J., Meffe G.K. \& Carroll C.R. (2005): Principles of conservation biology, 3rd edition. Sinauer Assoc. Inc. Sunderland, Mass., 699 pp.

Hanski I. \& Gaggiotti O. eds. (2004): Ecology, genetics and evolution of metapopulations. Elsevier/Academic Press London, 696 pp. + xix. 
Heller N.E. \& Zavaleta E. (2009): Biodiversity management in the face of climate change: A review of 22 years of recommendation. Biol. Conserv. 142: 14-32.

Hilty J.A., Lidicker jr. W.Z. \& Merenlender A.M. (2006): Corridor ecology: The science and practice of linking landscapes for biodiversity conservation. Island Press Washington, D.C., 324 pp.

Hobbs R.J. \& Suding K.N. eds. (2009): New models for ecosystem dynamics and restoration. Island Press Washington, D.C., 366 pp.

Holling C.S. ed. (1978): Adaptive environmental assessment and management. John Wiley and Sons Chicester, 377 pp.

Mackovc in P. (2000): A multi-level ecological network in the Czech Republic. Implementing the Territorial System of Ecological stability. GeoJournal 51: 211-220.

Mackovc`in P., Petr`íe`k V. \& Plesník J. (2005): Ecological networks in the Czech Republic. Commissioner General Office for the Participation of the Czech Republic at the World Exposition EXPO 2005 (Aichi, Japan) Prague, 46 pp.

Margules Ch. \& Sarkar S. (2007): Systematic conservation planning. Cambridge University Press Cambridge, 270 pp. + vii.

Mawdsley J.R., O'Malley R. \& Ojima D.S. (2009): A review of climate-change adaptation strategies for wildlife management and biodiversity conservation. Conserv. Biol. DOI: 10.1111/j.1523-1739.2009.01264.x.

Miko L. \& Zaunberger K. (2009): Biodiversity and climate change in the European Union. Ochrana pr írody 64, spec. issue: 20-24.

Ministry of the Environment of the Czech Republic (2004a): State Environmental Policy of the Czech Republic. 2004-2010. Ministry of the Environment of the Czech Republic, $60 \mathrm{pp}$.

Ministry of the Environment of the Czech Republic (2004b): National Programme to Abate the Climate Change Impacts in the Czech Republic. Ministry of the Environment of the Czech Republic Prague, $104 \mathrm{pp}$.

Ministry of the Environment of the Czech Republic (2005): National Biodiversity Strategy of the Czech Republic. Ministry of the Environment of the Czech Republic Prague, 137 pp. + cxxix.

Ministry of the Environment of the Czech Republic (2009a): Climate Protection Policy in the Czech Republic. Draft. Ministry of the Environment of the Czech Republic Prague, $146 \mathrm{pp}$.

Ministry of the Environment of the Czech Republic (2009b): Fourth National Report of the Czech Republic to Convention on Biological Diversity. Ministry of the Environment of the Czech Republic Prague, $92 \mathrm{pp}$.

Mlčoch S., Hošek J. \& Pelc F. eds. (1998): State Nature Conservation and Landscape Protection Programme of the Czech Republic. Ministry of the Environment of the Czech Republic, 21 pp. + xxi.

Naeem S., Bunker D.E., Hector A., Loreau M. \& Perrings Ch. (2009): Biodiversity, ecosystem functioning and human wellbeing: An ecological and economic perspective. Oxford University Press Oxford, 384 pp.

\section{$344 \quad$ (4): $547-555$.}


Office of the Government of the Czech Republic (2004): The Czech Republic Strategy for Sustainable Development. Office of the Government of the Czech Republic Prague, $63 \mathrm{pp}$.

Pelc F. ed. (2009): Strategy of the Ministry of the Environment of the Czech Republic on the adaptation measures in the landscape in connection with climate change. Unpublished manuscript. Ministry of the Environment of the Czech Republic Prague, 56 pp. + ix. (In Czech).

Pickett S.T.A., Ostfeld R.S., Shacha K.M. \& Likens G.E. eds. (1997): The ecological basis of conservation. Heterogeneity, ecosystems and biodiversity. Chapman \& Hall New York London, 466 pp.

Pickett S.T.A., Parker V.T. \& Fiedler P.L. (1992): The new paradigm in ecology: Implications for conservation biology above the species level. In Fiedler P.L. \& Jain S.K. (eds.): Conservation biology: the theory and practice of nature conservation, preservation, and management. Chapman \& Hall London New York: 65-88.

Pielke R.A. Jr. (2002): Policy, politics and perspective, Nature 416: 367-368.

Plesník J. (2004): Biological diversity in the Czech Republic: current state, main problems and prospects. In Secretariat of the Convention on Biological Diversity: Biological diversity on the Earth: state and prospects. Scientia Prague: 197-261 (In Czech).

Plesník J. (2008): Interaction between policy concerning spatial planning and ecological networks in Europe. Czech Republic-National Report. Agency for Nature Conservation and Landscape Protection of the Czech Republic Prague and European Centre for Nature Conservation Tilburg, the Netherlands, 41 pp.

Plesník J. \& Pelc F. (2009): Nature and the landscape in the Czech Republic and their adaptation to climate changes. Ochrana pr ${ }^{2}$ írody 64, spec. issue: 30-34.

Plesník J. \& Stan`ková J. eds. (2001): Status of biological resources and implementation of the Convention on Biological Diversity in the Czech Republic. First report. Ministry of the Environment of the Czech Republic Prague, $72 \mathrm{pp}$.

Pretel J. (2009): Current trends in the climate and projections for the future. Ochrana pr írody 64, spec. issue.: 2-7.

Rohde K. (2006): Nonequilibrium ecology. Cambridge University Press Cambridge, $236 \mathrm{pp}$.

Shafer C.L. (1995): Values and shortcomigs of small reserves. BioScience 45: 80-88.

Schröter D., Cramer W., Leemans R., Prentice I.C., Araújo M.B. et al. (2005): Ecosystem service supply and vulnerability to global change in Europe. Science 310: 1333-1337.

Schwartz M.W. (1999): Choosing the appropriate scale of reserves for conservation. Ann.Rev. Ecol. Syst. 30: 83-108.

Stern N. N. (2006): The economics of climate change: the Stern review. Cambridge University Press Cambridge, 712 pp.

Un (1994): Convention on Biological diversity. Text with annexes. United Nations New York, 17 pp.

Unep (2009): Draft findings of the Ad hoc Technical Expert Group on Bioodiversity and Climate Change. Secretariat of the Convention on Biological Diversity Montreal, 100 pp. 
WalterS S.C. (1986): Adaptive management of renewable resources. Mc Graww-Hill New York, 374 pp.

Wilson E.O. ed. (1988). Biodiversity. National Academy Press Washington, D.C., 521 pp.

World Bank (2009): Convenient solutions to sn inconvenient truth: Ecosystem-based approaches to climate change. World Bank Washington, D.C., 91 pp.

Wri/lucn/Unep (1992). Global biodiversity strategy. Guidelines for action to save, study, and use Earth's biotic wealth sustainably and equitably. World Resources Institute Washington. D.C., IUCN-The World Conservation Union Gland, Switzerland and United Nations Environment Programme Nairobi, 244 pp. 
ANEXO 1

BIODIVERSIDAD: ¿ENIGMA O TRIVIALIDAD?

La relativamente compleja naturaleza de la diversidad biológica (biodiversidad) se refleja en el hecho de que actualmente existen aproximadamente unas 40 propuestas de definición de aquélla. De acuerdo con la Convención sobre Diversidad Biológica ( $C B D$ ) diversidad biológica significa la variabilidad de organismos vivos de cualquier fuente, incluidos, entre otras cosas, los ecosistemas terrestres y marinos y otros ecosistemas acuáticos y los complejos ecológicos de los que forman parte; comprende la diversidad dentro de cada especie, entre las especies y de los ecosistemas (NU 1994). La biodiversidad se define asimismo con frecuencia como el total de diversidad y variabilidad de los organismos vivientes y de los sistemas de los que forman parte (WRI/IUN/UNEP 1992). Cubre el todo el ámbito de variación y variabilidad existente en los sistemas y organismos. En otras palabras, la biodiversidad es la variabilidad de la vida en todas sus formas, niveles y combinaciones. No consiste en la suma de todos los ecosistemas, especies y genética material. Por tanto, el término biodiversidad es una cualidad de la vida.

En todo caso, desde el final de los ochenta, cuando el conocido biólogo americano Edward Wilson y sus colegas formularon el concepto de biodiversidad, son tres los niveles o componentes más importantes de aquélla:

a) Genes/individuos

b) Población/especies

c) Comunidades/ecosistemas/paisaje

En el artículo seguimos las definiciones referidas de diversidad biológica. 
ANEXO 2

\section{LA NATURALEZA Y EL PAISAJE EN LA REPÚBLICA CHECA SE PRESENTAN BREVEMENTE}

El estado actual de la naturaleza y el paisaje en la República Checa se encuentra determinado por numerosos factores. Entre ellos está incluida la ubicación geográfica del país, el relieve específico, los sustratos rocosos extraordinariamente diversificados, las condiciones climáticas y el desarrollo en el último periodo interglaciar, que esta parte del continente europeo ha sufrido y sigue sufriendo todavía. Al igual que otras zonas de la Tierra en el siglo pasado, el ser humano ha sido, con mucho, el causante de los mayores efectos en las condiciones, cambios y tendencias en la naturaleza y el paisaje.

En lo que a categorías de uso básico del terreno se refiere, el 54,1\% del total del territorio lo compone el terreno agrícola, del cual las tres cuartas partes son tierras destinadas a cultivo temporal o permanente (el $38,1 \%$ del total del territorio de la República Checa, en 2008), siendo la mayor parte del resto del terreno agrícola (el 15,3\%) terreno no cultivable (praderas y pastos). El alto porcentaje de terreno cultivable supone una amenaza para el mantenimiento de la biodiversidad en las tierras de labranza. Los bosques, de distinta calidad medioambiental, representan la tercera parte del terreno del país. Las aguas cubren un $2 \%$ de la superficie, mientras que el $1,7 \%$ del territorio del país ha sido objeto de construcción. El resto lo constituyen tierras de labranza abandonadas, infraestructuras de transporte, zonas industriales, etc.

Desde el punto de vista de la biogeografía, el país se sitúa en el mismo centro de Europa: por ello el territorio de la República Checa se encuentra situado en cuatro sub-provincias biogeográficas. La mayor parte del territorio se localiza en la provincia biogeográfica de las tierras altas centroeuropeas, comprendiendo dos sub-provincias (la herciniana en Bohemia y el oeste de Moravia y la cárpata en el este de Moravia). Debido a su posición única, en el periodo cuaternario el territorio de la actual República Checa se situaba en la frontera de las glaciaciones continental y alpina. Por tanto, era un cruce norte-sur y este-oeste de Europa, resultado de lo cual son las importantísimas rutas de migración continental de biota, por ej. algunas de las principales rutas de aves migratorias euroasiáticas.

A pesar del insatisfactorio estado general en que se encuentra la naturaleza, claro ejemplo de lo cual es el Triángulo Negro, en la República Checa se han conservado partes valiosas de la naturaleza en un estado relativamente bueno, o que permite el restablecimiento de los procesos naturales básicos.

La composición geológica diversa y la geomorfología, junto a la diversidad de condiciones geográficas y climáticas, dan lugar en la República Checa a una diversidad biológica y de paisaje relativamente alta, a pesar de su tamaño reducido. En aquélla es posible encontrar el siguiente número de especies: 391 especies de verte-

348 brados, entre 30.600 y 50.000 especies de invertebrados (de ellas, de 24.800 a 43.000 
especies de insectos), 2.700 especies de plantas vasculares, 886 especies de briófitos (musgos), 1.534 especies de líquenes, aproximadamente 30.000 especies de hongos y entre 6.180 y 15.000 especies de cianobacterias y algas, en total, de 72.000 a 102.000 especies (virus y bacterias excluidos). La flora representa aproximadamente el $25 \%$ de las 11.000 especies conocidas de Europa (Plesník \& Staňková 2001, Plesník 2004, Ministerio de Medio Ambiente de la República Checa 2009b).

De acuerdo con las Listas Rojas actuales, elaboradas por la Agencia para la Conservación de la Naturaleza y Protección del Paisaje de la República Checa, aproximadamente el $19 \%$ de las especies de mamíferos, el 52\% de las especies de aves nidificantes, el $61 \%$ de las especies reptiles, el $60 \%$ de las especies anfibias, el $46 \%$ de las especies ictícolas, el $10 \%$ de las mariposas, el $60 \%$ de las plantas vasculares y el $25 \%$ de las especies de musgo, se encuentran hoy día en peligro de extinción en la República Checa. 
